\title{
Upregulation of MicroRNA-1246 Is Associated with BRAF Inhibitor Resistance in Melanoma Cells with Mutant BRAF
}

\author{
Jae-Hyeon Kim, MS ${ }^{1}$ \\ Jun-Ho Ahn, PhD² \\ Michael Lee, $\mathrm{PhD}^{1}$
}

\begin{abstract}
Purpose
Intrinsic and acquired resistance limit the therapeutic benefits of inhibitors of oncogenic BRAF in melanoma. To identify microRNAs (miRNAs) associated with resistance to a BRAF inhibitor, we compared miRNA expression levels in three cell lines with different BRAF inhibitor sensitivity.
\end{abstract}

\section{Materials and Methods}

miRNA microarray analysis was conducted to compare miRNA expression levels. Real-time quantitative reverse-transcription polymerase chain reaction (qRT-PCR) was performed to confirm the expression of differentially expressed miRNAs. The cellular effects of miR-1246 were further examined by MTT assay, immunoblotting analysis, cell cycle analysis, flow cytometric assay of apoptosis, and autophagy assay.

\section{Results}

The miRNA microarray analysis and qRT-PCR identified five miRNAs (miR-3617, miR-92a1, miR-1246, miR-193b-3p, and miR-17-3p) with expression that was consistently altered in two BRAF inhibitor-resistant cell lines. Among the five miRNAs, a miR-1246 mimic significantly reduced the antiproliferative effects of the BRAF inhibitor PLX4720 in BRAF inhibitorresistant A375P (A375P/Mdr) cells, suggesting that miR-1246 upregulation confers acquired resistance to BRAF inhibition. In particular, apoptosis was identified as a major type of cell death in miR-1246-transfected cells; however, necrosis predominated in mimiccontrol-transfected cells, indicating that the resistance to PLX4720 in miR-1246 mimictransfected cells is predominantly due to a reduction in necrosis. Furthermore, we found that miR-1246 promoted $\mathrm{G}_{2} / \mathrm{M}$ arrest through autophagy as a way to escape cell death by necrosis and apoptosis in response to PLX4720. The promotion of BRAF inhibitor resistance by miR-1246 was associated with lowered levels of $p$-ERK.

\section{Conclusion}

These results suggest that miR-1246 may be a potential therapeutic target in melanoma with acquired resistance to BRAF inhibitors.
Incheon National University, 119 Academy-ro

Yeonsu-gu, Incheon 22012, Korea

Tel: 82-32-835-8247

Fax: 82-32-835-0763

E-mail: mikelee@inu.ac.kr

Received July 2, 2016

Accepted December 19, 2016

Published Online January 3, 2017
Key words

MicroRNAs, BRAF, PLX 4720, Drug resistance, Melanoma, Microarray analysis

\section{Introduction}

$B R A F$ somatic mutations that render BRAF constitutively active are observed in 50\%-60\% of malignant melanomas [1]. Thus, BRAF inhibitors have recently shown promise for the treatment of metastatic melanoma harboring such $B R A F$ mutations [2]. We also reported UAI-201 (also known as UI-152) as a potent ATP-competitive inhibitor of RAF proteins [3]. UAI-201 is more than 1,000-fold more selective at inhibiting the proliferation of tumor cell lines bearing the $B R A F$ V600E mutation when compared with that of cells carrying wild-type $B R A F[3]$. However, the development of acquired resistance to inhibitors of oncogenic BRAF limits 
the duration of the tumor response [4]. Besides BRAF inhibitors, most anticancer drugs have the problem of drug resistance, which limits their effectiveness. Accordingly, understanding the molecular mechanisms of drug resistance is necessary to improve the effectiveness of cancer therapies. In general, reactivation of the mitogen-activated protein kinase (MAPK) pathway is considered a primary mechanism underlying the acquired resistance to BRAF inhibitors [5]. Our previous study indicated that induction of resistance to a BRAF inhibitor is associated with the inability of Spry2 to inhibit BRAF V600E activity in cells with mutant BRAF [6]. In fact, the relief of feedback after targeted therapy may be viewed as a key contributor to therapeutic resistance [7].

Small noncoding microRNAs (miRNAs) have been confirmed to regulate the expression of target mRNAs by repressing their translation [8]. A growing body of evidence shows that dysregulation of miRNA expression contributes to acquisition of drug resistance by cancer cells [9]. Nevertheless, relatively few studies have explored the roles of miRNAs in resistance to BRAF inhibitor therapy, although several studies identified miRNAs that alter some of the oncogenic factors in melanoma cells [10]. In particular, overexpression of miR-514a inhibits NF1 expression, which is correlated with increased survival of BRAF V600E cells treated with PLX4032 [11].

In this study, we used the Affymetrix miRNA V3.0 microarray profiling platform to compare miRNA expression levels in three melanoma cell lines: BRAF inhibitor-sensitive A375P BRAF V600E cells, their BRAF inhibitor-resistant counterparts (A375P/Mdr), and SK-MEL-2 BRAF wild type (WT) cells. The A375P/Mdr cells with acquired resistance to BRAF inhibitors were generated by propagating parental A375P cells harboring the BRAF V600E mutation at increasing concentrations of a BRAF inhibitor to implement chronic selection [12]. The SK-MEL-2 cell line expressing WT BRAF has intrinsic resistance to BRAF inhibition because the BRAF inhibitor lacks activity against cell lines that express WT BRAF. We found that 43 miRNAs are deregulated in BRAF inhibitor-resistant cells compared with those in BRAF inhibitor-sensitive cells. We also found that ectopically expressed miR-1246 can confer resistance to PLX4720 (a BRAF inhibitor) to A375P/Mdr cells.

\section{Materials and Methods}

\section{Materials}

The Affymetrix miRNA V3.0 array profiling platform was supplied by Affymetrix (Santa Clara, CA). The RNeasy Midi
Kit was acquired from Qiagen (Valencia, CA). SYBR Premix EX TaqII, which was used for real time polymerase chain reaction (PCR), was purchased from Takara Korea Biomedical Inc. (Seoul, Korea). For the apoptosis assay, a FITC Annexin V Apoptosis Detection Kit was purchased from BD Biosciences Pharmingen (San Diego, CA). For the flow cytometric autophagy assay, Cyto-ID Green dye was acquired from ENZO Life Sciences, Inc. (Farmingdale, NY). Rabbit polyclonal anti-MEK, anti-ERK, anti-p2 ${ }^{\text {Cip1 }}$, and anti-p27 ${ }^{\text {Kip1 }}$ antibodies were acquired from Santa Cruz Biotechnology (Santa Cruz, CA), whereas anti-phospho-MEK (anti-p-MEK Ser217/221) and anti-phospho-ERK (anti-p-ERK, Thr202/ Tyr204) antibodies were purchased from Cell Signaling Technology (Danvers, MA). Dulbecco's modified Eagle's medium (DMEM), fetal bovine serum (FBS), and penicillin-streptomycin were purchased from Life Technologies (Carlsbad, CA). The reagents for sodium dodecyl sulfate (SDS)-polyacrylamide gel electrophoresis were acquired from BioRad (Hercules, CA), while PLX4720 was obtained from Selleck Chemicals (Houston, TX).

\section{Cell lines and cell culture}

For this study, we used three melanoma cell lines: BRAF inhibitor-sensitive A375P BRAF V600E cells, their BRAF inhibitor-resistant counterparts (A375P/Mdr), and SK-MEL2 BRAF-WT cells with intrinsic resistance to the BRAF inhibitor. A375P and SK-MEL-2 cells were acquired from either the Korean Cell Line Bank (KCLB; Seoul, Korea) or YOUAI Co., Ltd. (Suwon, Korea). A375P/Mdr cells were previously established by chronic selection with increasing doses of an inhibitor of oncogenic BRAF [12]. All cell lines were maintained at $37^{\circ} \mathrm{C}$ in DMEM supplemented with $10 \%$ FBS, penicillin-streptomycin, and glutamine. A375P/Mdr cells were further propagated in growth medium containing the BRAF inhibitor PLX4720 $(1 \mu \mathrm{M})$. Before their use in the experiments, A375P/Mdr cells were maintained in PLX4720free culture medium and subcultured at least three times. For experimental purposes, the cells were cultured in $60-\mathrm{mm}$ tissue culture dishes until they reached $\sim 80 \%$ confluence. PLX4720 was dissolved in dimethyl sulfoxide (DMSO) and diluted immediately before each experiment. The DMSO concentration was less than $0.1 \%$ in all experiments.

\section{MiRNA microarray profiling}

Total RNA was extracted from three melanoma cell lines using a miRNeasy Kit (Qiagen). The quantity and purity of RNA samples were assessed using an Agilent 2100 Bioanalyzer (Agilent Technologies, Palo Alto, CA). Total RNA was subjected to miRNA expression profiling (miRBase release 17.0) using the Affymetrix miRNA V3.0 array with a probe 
set for 1,733 human mature miRNAs. Microarray hybridization, data generation, and normalization were performed by GenoCheck Co., Ltd. (Seoul, Korea). The Affymetrix Expression Console software and Affymetrix 7G scanner were used for bioinformatic analysis and visualization of microarray data.

\section{Real-time quantitative reverse-transcription PCR}

Real-time quantitative reverse-transcription PCR (qRTPCR) was performed to confirm the expression of differentially expressed miRNAs that were identified in the miRNA microarray analysis. Briefly, total RNA was isolated using a RNeasy Mini Kit (Qiagen). The primer sequences were then selected using the Primer3Plus software (http:// primer3plus. $\mathrm{com} /$ ), after which the primers were synthesized by Bioneer (Daejeon, Korea). The primers used in the present study had the following oligonucleotide sequences: miR-3617, $5^{\prime}$-CTCAAAGGGCTCATTGGTTG- $3^{\prime}$ and $5^{\prime}$-GCCTGCTAAATGGAGTGTGC-3'; miR-92a-1, 5'-GCCCAATCAAACTGTCCTGT- $3^{\prime}$ and $5^{\prime}$-CAATCCCCACCAAACTCAAC- $3^{\prime}$; miR-1246, 5'-TGAAGTAGGACTGGGCAGAGA- ${ }^{\prime}$ and $5^{\prime}$-TGTTTGCAATAGCCCTTTGAG-3'; miR-193b-3p, 5'-ATCCCGGTTCTCCAAACTCT- $3^{\prime}$ and $5^{\prime}$-TGGTAGCTCTCTGCCCTCAC- $3^{\prime}$; miR-17-3p, $5^{\prime}$-TTGTAAAACTGAAGATTGTGACCA-3' and 5'-TGCCAGAAGGAGCACTTAGG-3'. qRT-PCR was conducted on an Applied Biosystems 7300 Real-Time PCR System (Applied Biosystems, Foster City, CA) using SYBR Premix EX TaqII. The mean threshold cycle $\left(C_{t}\right)$ was calculated from data from triplicate reactions. The $2^{-\Delta \Delta C t}$ method was used to calculate the fold differences in miRNA among the tested samples [13].

\section{5. miRNA mimic transfection procedures}

The sequence of the miR-1246 mimic ( $5^{\prime}$-AAUGGAUUUUUGGAGCAGG-3') was retrieved from the miRBase database (http://www.mirbase.org), and the sequence of the miR-122 inhibitor was completely complementary to that of miR-1246. Cells were transiently transfected with $50 \mathrm{nM} \mathrm{miR-}$ 1246 mimic, inhibitor or its negative control (Bioneer) using Lipofectamine 2000 (Invitrogen, Carlsbad, CA).

\section{Cell growth assay}

Cells were transfected with either miRNA mimics or inhibitors and their control using Lipofectamine 2000 for 24 hours. The cells were plated in quadruplicate in 96-well microtiter plates (Costar, Cambridge, MA) at $5 \times 10^{3} /$ well and then incubated with $\mathrm{PLX} 4720$ at $37^{\circ} \mathrm{C}$ in a humidified $5 \% \mathrm{CO}_{2}$ incubator. On day 3 , the cells were incubated with 3-(4,5dimethylthiazol-2-yl)-2,5-diphenyltetrazolium bromide (MTT) at $37^{\circ} \mathrm{C}$ for 3 hours. The absorbance of the samples against a background control (medium alone), which served as a blank, was measured at $450 \mathrm{~nm}$ using a SpectraMax 190 microplate reader (Molecular Devices, Sunnyvale, CA).

\section{Flow cytometric analysis of autophagic activity with Cyto-ID staining}

The Cyto-ID fluorescent reagent specifically labels autophagic vacuoles and colocalizes with LC3 [14]. Thus, autophagosome formation in cells was detected using the Cyto-ID Autophagy Detection Kit (ENZO Life Sciences Inc.). Briefly, cells were trypsinized, pelleted by centrifugation, and washed twice in a phenol-red-free culture medium with $2 \%$ FBS. The cells were then resuspended in $0.5 \mathrm{~mL}$ of freshly diluted Cyto-ID reagent and incubated at $37^{\circ} \mathrm{C}$ for 30 minutes, after which the Cyto-ID fluorescence of cells was immediately analyzed using a Gallios flow cytometer and the Kaluza analysis software (Beckman Coulter Inc., Brea, CA). The percentage of cells with Cyto-ID staining was used to assess the formation of autophagosomes.

\section{Apoptosis assay using annexin $\mathrm{V}$ staining and flow cytometry}

Apoptosis was quantified using the FITC Annexin V Apoptosis Detection Kit (BD Biosciences Pharmingen). Briefly, after treatment with the BRAF inhibitor, $2 \times 10^{6}$ cells were harvested, washed with ice-cold phosphate-buffered saline (PBS), resuspended in $200 \mu \mathrm{L}$ of binding buffer [ $10 \mathrm{mM}$ 4-(2-hydroxyethyl)-1-piperazineethanesulfonic acid (HEPES)$\mathrm{NaOH}$ pH 7.4, $140 \mathrm{mM} \mathrm{NaCl}$, and $2.5 \mathrm{mM} \mathrm{CaCl}_{2}$, and incubated with $5 \mu \mathrm{L}$ of annexin $\mathrm{V}$ conjugated with FITC for 10 minutes at room temperature in the dark. The samples were then washed with binding buffer, resuspended in PBS, counterstained with propidium iodide (PI), and analyzed on the Gallios flow cytometer and with the Kaluza analysis software. The annexin $\mathrm{V}^{-} / \mathrm{PI}^{+}$cells were assumed to be necrotic, annexin $\mathrm{V}^{+} / \mathrm{PI}^{+}$cells were considered late apoptotic, and annexin $\mathrm{V}^{+} / \mathrm{PI}^{-}$cells were assumed to be early apoptotic.

\section{Preparation of cell lysates and immunoblot analysis}

Whole-cell lysates were prepared as follows. Cells were washed twice with ice-cold PBS, then harvested by scraping into lysis buffer (20 mM Tris- $\mathrm{HCl} \mathrm{pH}$ 8.0, $150 \mathrm{mM} \mathrm{NaCl}, 1 \%$ Triton X-100, 2 mM EDTA, $1 \mathrm{mM}$ phenylmethylsulfonyl fluoride, $20 \mu \mathrm{g} / \mathrm{mL}$ aprotinin, $10 \mu \mathrm{g} / \mathrm{mL}$ leupeptin, $20 \mathrm{mM}$ $\beta$-glycerophosphate, and $2 \mathrm{mM}$ sodium fluoride). Cell lysates were clarified by centrifugation at $15,000 \times \mathrm{g}$ for 10 minutes at $4^{\circ} \mathrm{C}$, after which lysate protein concentrations were determined with the BCA Protein Assay Reagent Kit (Pierce, 
Table 1. List of miRNA analyzed by real-time quantitative reverse-transcription polymerase chain reaction

\begin{tabular}{|c|c|c|c|}
\hline \multirow{2}{*}{ miRNA name } & \multicolumn{2}{|c|}{ Fold change vs. A375P } & \multirow{2}{*}{ miRNA sequence } \\
\hline & SK-MEL-2 & A375P/Mdr & \\
\hline \multicolumn{4}{|l|}{ Up-regulation } \\
\hline hsa-miR-1246 & 3.89 & 3.78 & AAUGGAUUUUUGGAGCAGG \\
\hline hsa-miR-146b-5p & 19.40 & 3.10 & UGAGAACUGAAUUCCAUAGGCU \\
\hline hsa-miR-1909-3p & 2.53 & 4.17 & CGCAGGGGCCGGGUGCUCACCG \\
\hline hsa-miR-193b-3p & 4.24 & 2.25 & AACUGGCCCUCAAAGUCCCGCU \\
\hline hsa-miR-1972 & 4.32 & 2.46 & UCAGGCCAGGCACAGUGGCUCA \\
\hline hsa-miR-1973 & 3.35 & 3.48 & ACCGUGCAAAGGUAGCAUA \\
\hline hsa-miR-3195 & 6.31 & 6.18 & CGCGCCGGGCCCGGGUU \\
\hline hsa-miR-3617 & 6.48 & 2.64 & AAAGACAUAGUUGCAAGAUGGG \\
\hline hsa-miR-4532 & 4.18 & 8.63 & CCCCGGGGAGCCCGGCG \\
\hline hsa-miR-4726-5p & 4.06 & 5.75 & AGGGCCAGAGGAGCCUGGAGUGG \\
\hline \multicolumn{4}{|l|}{ Down-regulation } \\
\hline hsa-miR-1184 & 2.77 & 2.77 & CCUGCAGCGACUUGAUGGCUUCC \\
\hline hsa-miR-125b-1-5p & 8.98 & 2.20 & ACGGGUUAGGCUCUUGGGAGCU \\
\hline hsa-miR-135b-3p & 2.87 & 2.60 & AUGUAGGGCUAAAAGCCAUGGG \\
\hline hsa-miR-139b-5p & 2.58 & 5.86 & UCUACAGUGCACGUGUCUCCAG \\
\hline hsa-miR-17-3p & 2.98 & 2.22 & ACUGCAGUGAAGGCACUUGUAG \\
\hline hsa-miR-199a-3p & 15.91 & 19.26 & ACAGUAGUCUGCACAUUGGUUA \\
\hline hsa-miR-199b-3p & 15.82 & 31.95 & ACAGUAGUCUGCACAUUGGUUA \\
\hline hsa-miR-20b-3p & 2.01 & 3.01 & ACUGUAGUAUGGGCACUUCCAG \\
\hline hsa-miR-222-5p & 2.37 & 6.75 & CUCAGUAGCCAGUGUAGAUCCU \\
\hline hsa-miR-23a-5p & 2.99 & 2.40 & GGGGUUCCUGGGGAUGGGAUUU \\
\hline hsa-miR-33b-3p & 6.52 & 2.56 & CAGUGCCUCGGCAGUGCAGCCC \\
\hline hsa-miR-92a-1-5p & 6.78 & 6.31 & AGGUUGGGAUCGGUUGCAAUGCU \\
\hline
\end{tabular}

Rockford, IL). For immunoblotting, whole-cell lysates were denatured in Laemmli sample buffer, then resolved by SDSpolyacrylamide gel electrophoresis. The proteins were transferred to a nitrocellulose membrane, after which immunoblotting analysis was performed using the appropriate primary antibodies. The immune complexes were visualized with an Amersham ECL Kit (GE Healthcare Life Sciences) in the dark and images were captured using the BioRad ChemiDoc XRS+ instrument (Hercules, CA). Band intensity values were measured using the BioRad Image Lab software ver. 5.2.1 (BioRad Laboratories Inc.).

\section{Results}

\section{TaqMan qRT-PCR confirmation of differentially regu- lated miRNAs}

To determine which miRNAs are responsible for resistance to BRAF inhibitors, we examined changes in miRNA expression in three cell lines: BRAF inhibitor-sensitive A375P BRAF V600E cells and two BRAF inhibitor-resistant cell lines (A375P/Mdr and SK-MEL-2 BRAF-WT). The miRNAs with an at least two-fold change in expression between BRAF inhibitor-sensitive and -resistant cell lines were identified as differentially expressed. Averaged intensity measurements identified 43 miRNAs with expression that was consistently altered two-fold or more in two BRAF inhibitor-resistant cell lines, regardless of whether the resistance was intrinsic or acquired. Of these, 25 miRNAs were consistently upregulated and 18 were downregulated by more than two-fold.

To validate the results of the microarray analysis, qRT-PCR was conducted for the 22 selected miRNAs (10 upregulated and 12 downregulated miRNAs) using the TaqMan MicroRNA Assay (Table 1). Some discrepancies were detected when miRNA microarray data were collated with qRT-PCR-measured expression levels. Nevertheless, qRTPCR analysis of five miRNAs showed excellent agreement with the microarray experiments (Fig. 1). We found miR1246, miR-193b-3p, and miR-3617 to be consistently upregu- 
miR-17-3p
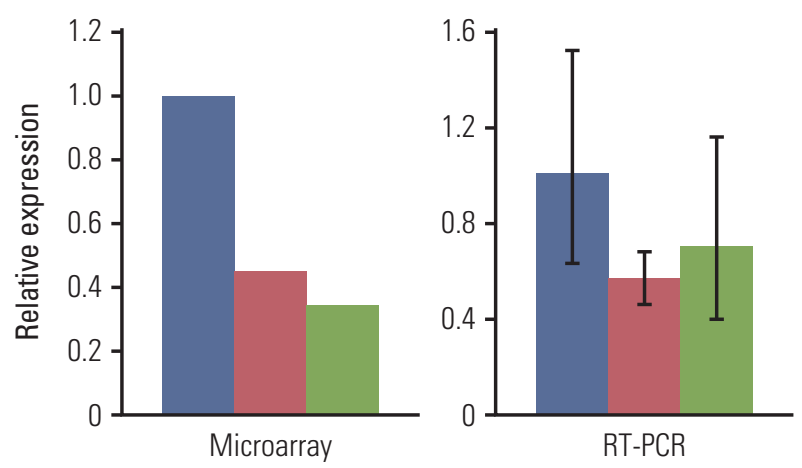

miR-3617
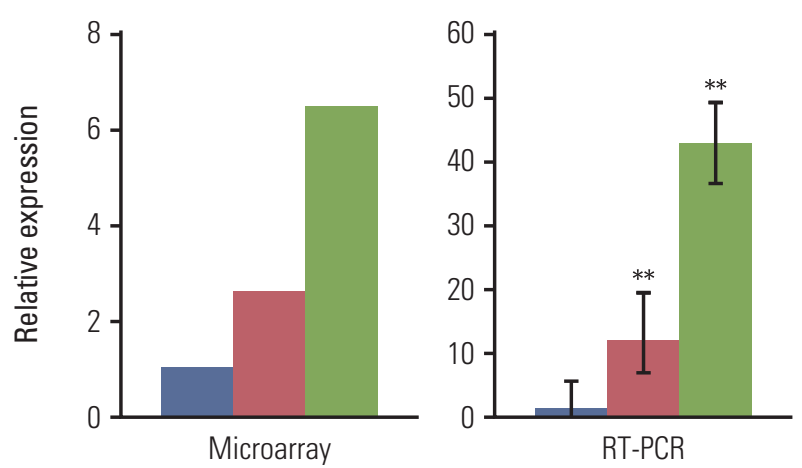

miR-193b-3p

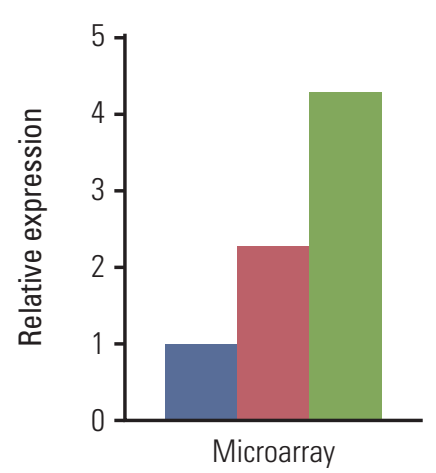

miR-92a-1
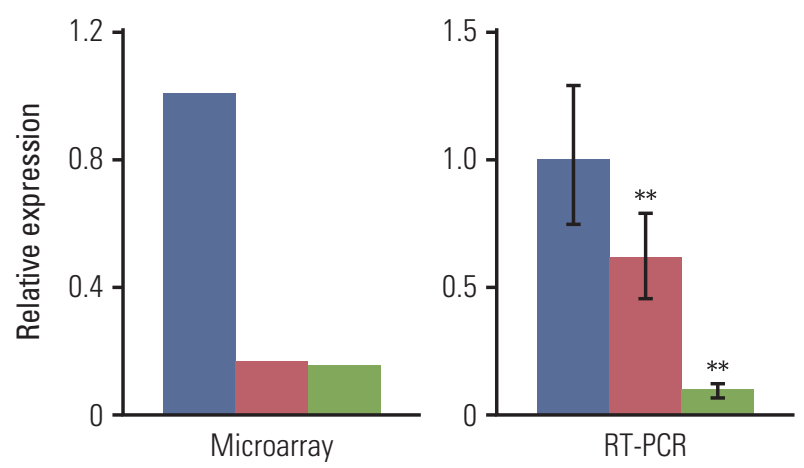

$\operatorname{miR}-1246$
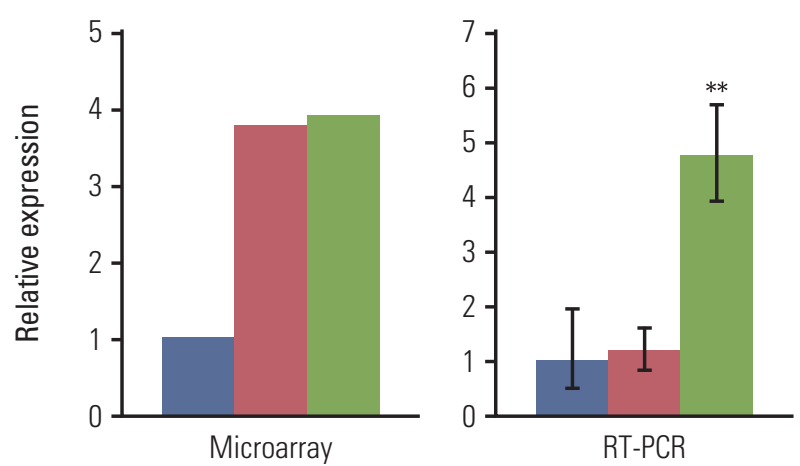

Fig. 1. Validation of miRNA microarray data using miRNA-specific real-time quantitative reverse-transcription polymerase chain reaction. Real-time reverse-transcription polymerase chain reaction was conducted to measure the mRNA expression levels of miR-17-3-p, miR-92a-1, miR-3617, miR-1246, and miR-193b-3p. The mean threshold cycle $\left(C_{t}\right)$ was determined based on triplicate reactions. The $2^{-\Delta \Delta C t}$ method was used to calculate the fold differences in miRNA expression among the tested samples. The expression of the target genes was normalized to GAPDH expression. ${ }^{* *} \mathrm{p}<0.01$ according to Dunnett's t test in relative to BRAF inhibitor-sensitive A375P cells. 


\section{A375P}
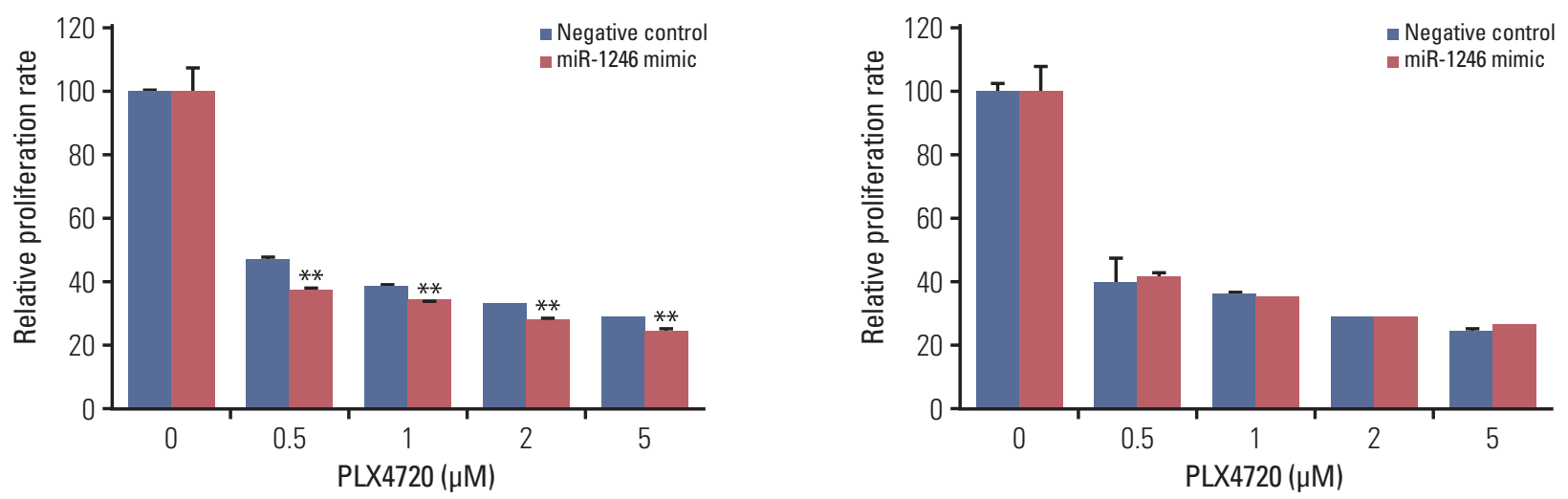

B

A375P/Mdr
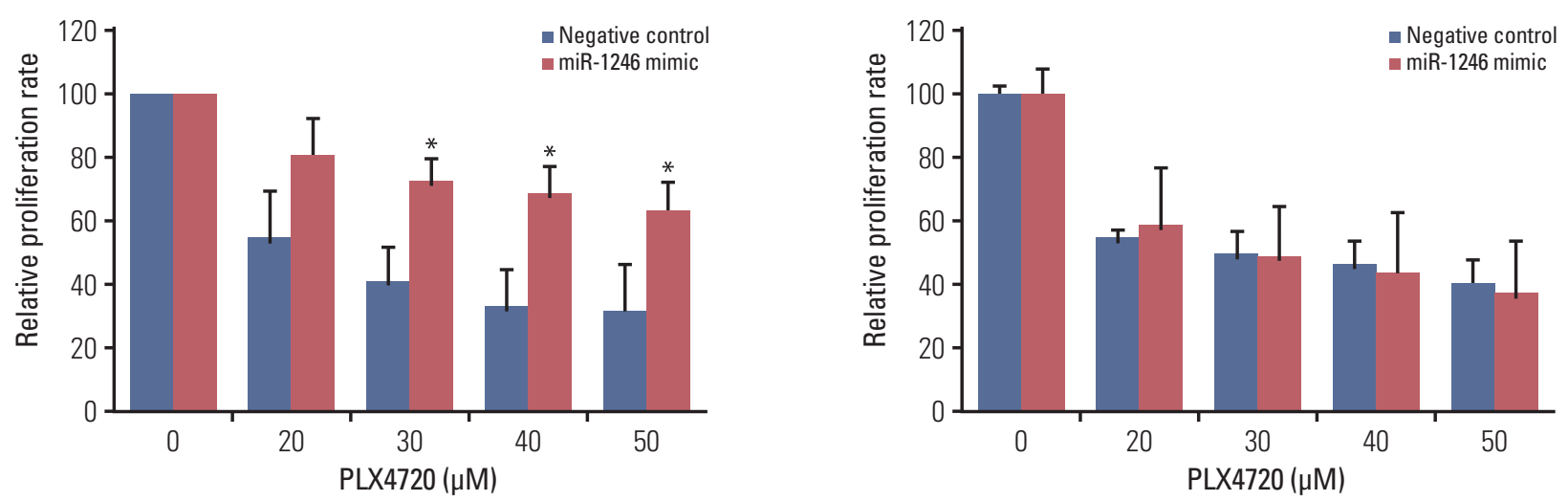

SK-MEL-2
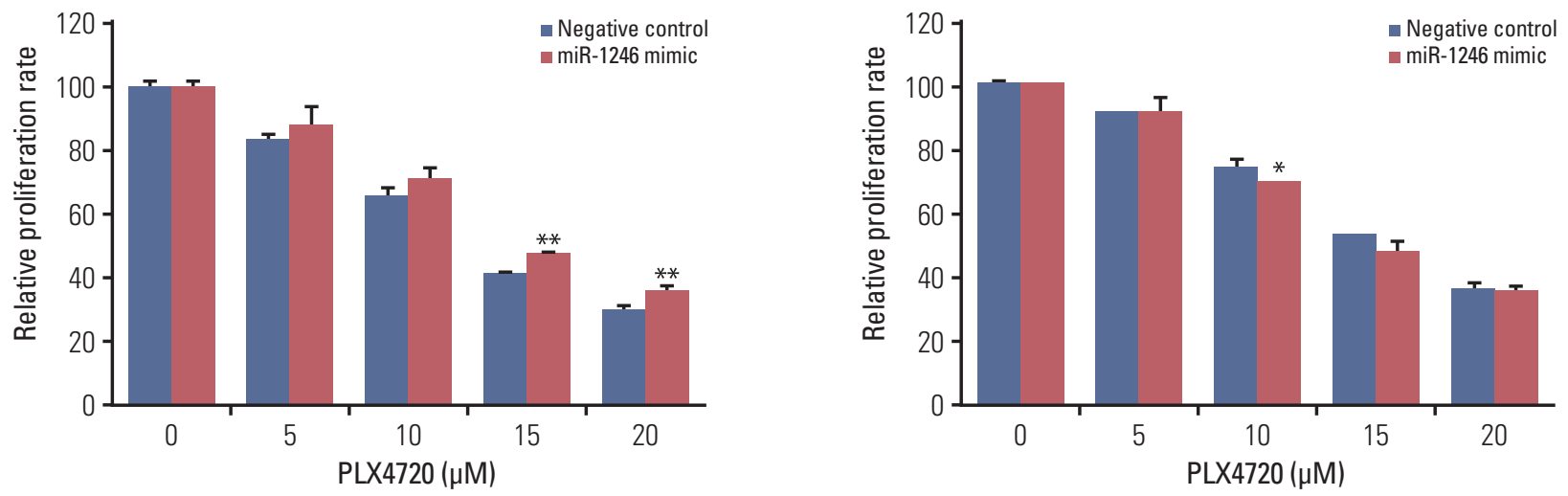

Fig. 2. MicroRNA-1246 controls the resistance to the BRAF inhibitor in melanoma cells. A375P (A), A375P/Mdr (B), and SK-MEL-2 (C) cells were transiently transfected with a miR-1246 mimic or inhibitor for 24 hours and then treated with PLX4720 in 96-well plates for 3 days. Cell growth was then evaluated using a MTT assay. The data represent the means (standard deviation) of quadruplicates from one of three representative experiments $(\mathrm{A}, \mathrm{C})$ or of three independent experiments (B). ${ }^{* *} \mathrm{p}<0.01$ and ${ }^{*} \mathrm{p}<0.05$ compared with the negative control, according to Dunnett's t test. 


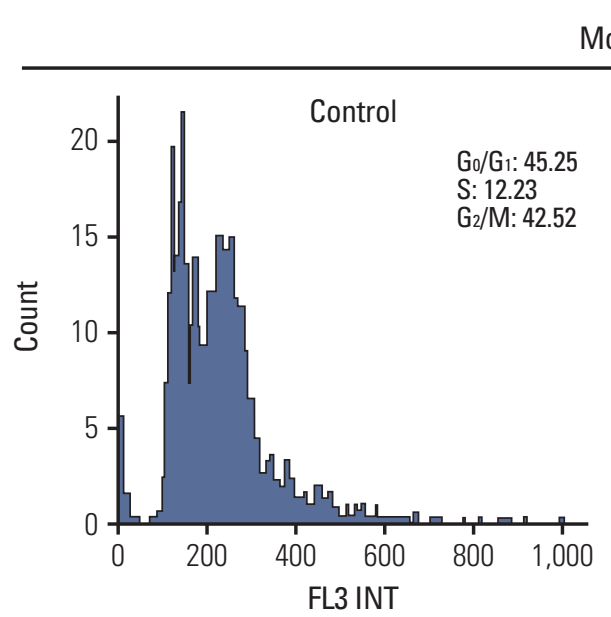

Mock control

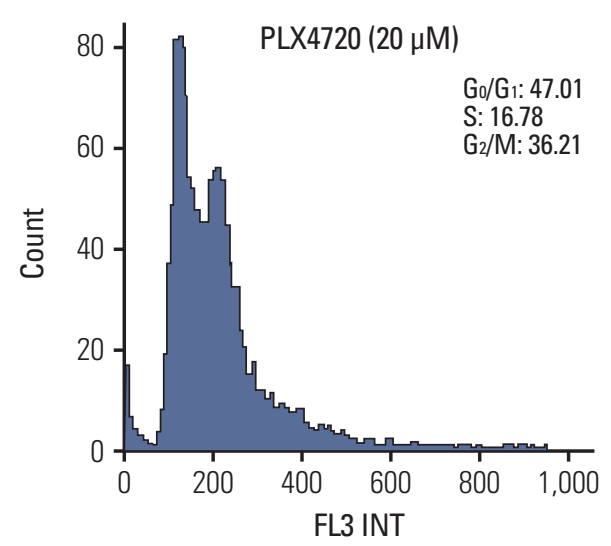

miR-1246 mimic
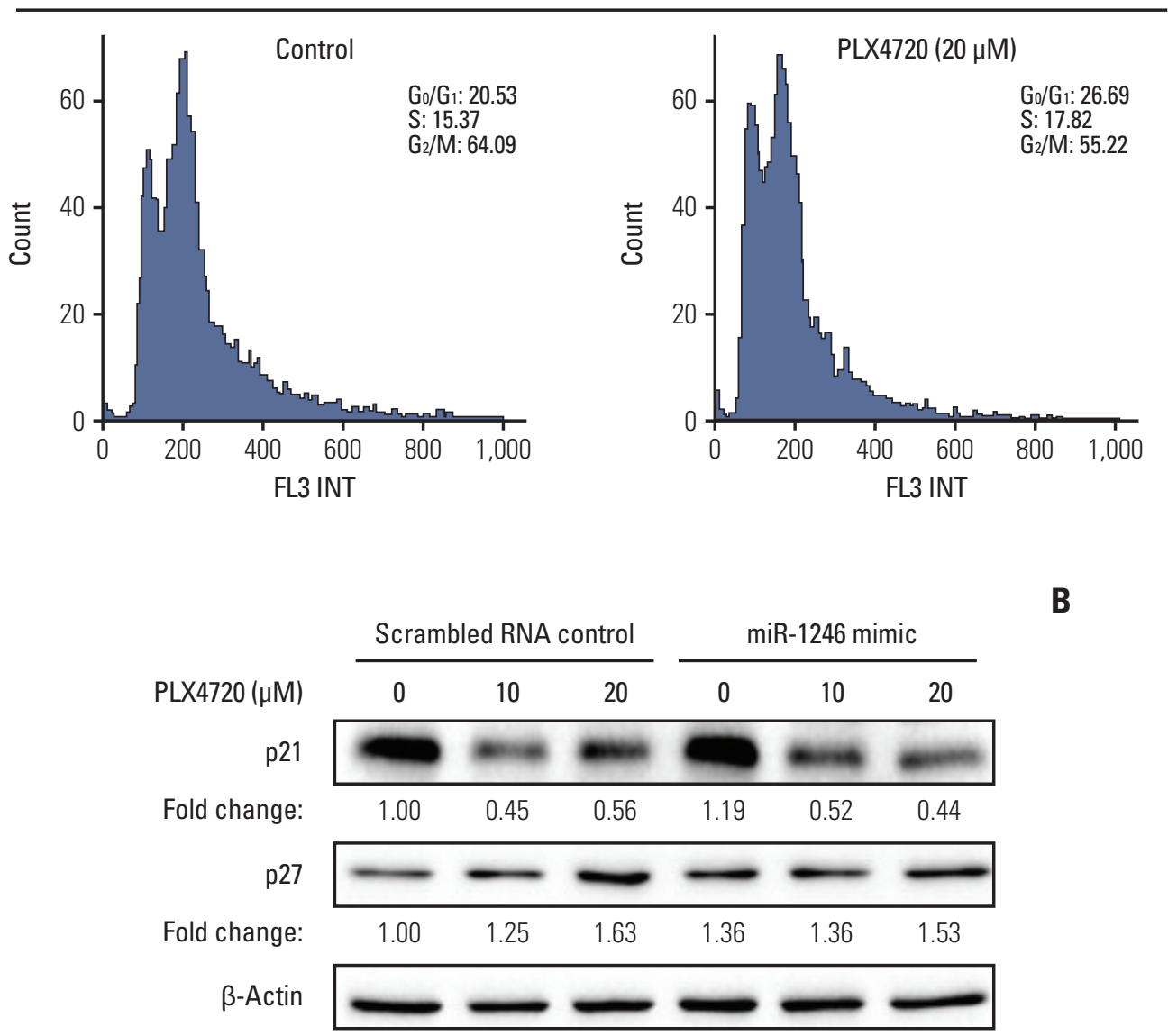

Fig. 3. Transfection with miR-1246 leads to decreased cell growth and $\mathrm{G}_{2} / \mathrm{M}$ arrest. A375P/Mdr cells were transiently transfected with miR-1246 mimic or control RNA for 24 hours, then treated with PLX4720 for 48 hours (A, B) or 72 hours (C). (A) Cell cycle progression was assessed by staining fixed cells with propidium iodide and by flow cytometric analysis (with proper preparation of the cells). The vertical and horizontal axes represent the cell number and DNA fluorescence intensity, respectively. The percentages of cells in individual cell cycle phases were quantified using the Cell Quest Pro software. The presented results are representative of at least three independent experiments. (B) Whole cell extracts were prepared at 48 hours after PLX4720 treatment. The expression levels of p21 ${ }^{\mathrm{Cip} 1}$ and p27 ${ }^{\mathrm{Kip} 1}$ were assessed by immunoblotting. $\beta$-Actin was assessed as a control for protein loading. The results presented are representative of two independent experiments. (Continued to the next page) 


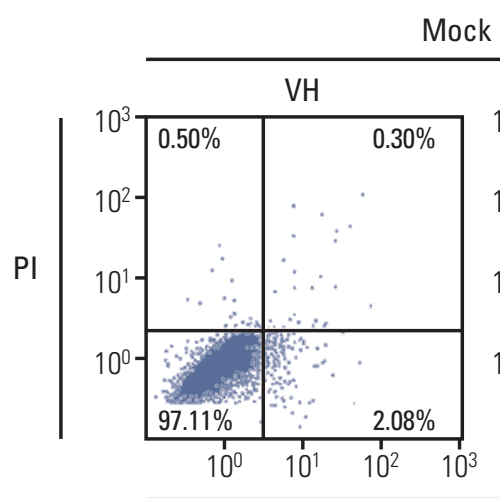

Mock control
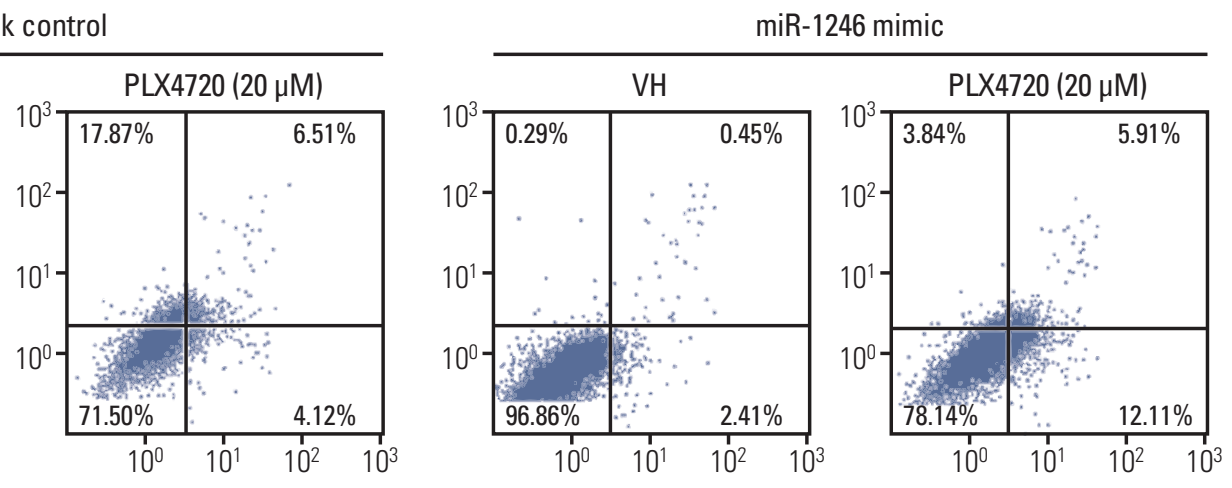

Annexin V

Fig. 3. (Continued from the previous page) (C) Apoptosis was evaluated by staining with annexin $\mathrm{V} 72$ hours after transfection. The flow cytometric profile shows the annexin V-FITC staining on the X-axis and the propidium iodide (PI) staining on the $\mathrm{Y}$-axis. The numbers represent the percentage of cells in each quadrant. The presented results are representative of at least three independent experiments.

lated in the two BRAF inhibitor-resistant cell lines. In contrast, miR-17-3p and miR-92a-1 were downregulated in the two BRAF inhibitor-resistant cell lines.

\section{The miR-1246 mimic confers resistance to oncogenic BRAF inhibitors in A375P/Mdr cells}

To explore the cellular functions of miRNAs associated with resistance to BRAF inhibitors, the effects of miRNAs on cell proliferation were determined after transient transfection (Fig. 2). Synthetic oligonucleotide miRNA mimics and inhibitors were transfected into three cell lines, and proliferation was quantified by a colorimetric assay. Of the five miRNAs tested (S1 Fig.), miR-1246 (which was upregulated in BRAF inhibitor-resistant cells) conferred strong additional PLX4720 resistance to A375P/Mdr cells (Fig. 2B), implying that miR-1246 upregulation is responsible for resistance to BRAF inhibition. In addition, in SK-MEL-2 cells (intrinsically resistant to BRAF inhibition), the difference in cell growth rate between mimic-control-transfected and miR-1246 mimic-transfected cells was statistically significant; however, the efficacy of miR-1246 was modest (Fig. 2C). MiR-1246 did not cause PLX4720 resistance in BRAF inhibitor-sensitive A375P cells. Moreover, miR-1246 inhibitors showed negligible effects on PLX4720-induced growth inhibition relative to the mimic control.

\section{Effects of miR-1246 on PLX4720-induced $\mathrm{G}_{2} / \mathrm{M}$ arrest and apoptosis}

To characterize the mechanism by which miR-1246 induces BRAF inhibitor resistance to A375P/Mdr cells, the effect of miR-1246 on cell cycle distribution was determined by flow cytometry. Cell cycle analysis showed a significant decrease in the cell population with $2 \mathrm{~N}$ DNA content (G1: $45.25 \%$ in mimic-control-transfected population to $20.53 \%$ in population transfected with the miR-1246 mimic), whereas a marked increase was observed in the population with $4 \mathrm{~N}$ DNA content $\left(\mathrm{G}_{2} / \mathrm{M}: 42.52 \%\right.$ in mimic-control-transfected population to $64.09 \%$ in population transfected with the miR1246 mimic) (Fig. 3A). The number of cells in the $S$ phase was approximately unchanged $(12.23 \%$ vs. $15.37 \%)$. We also investigated the expression of the cyclin-dependent kinase inhibitors (CKIs) p21 $1^{\mathrm{Cip} 1}$ and p27 $7^{\mathrm{Kip} 1}$ (Fig. 3B). We detected a remarkable decrease of $\mathrm{p} 21^{\mathrm{Cip} 1}$ in $\mathrm{A} 375 \mathrm{P} / \mathrm{Mdr}$ cells after PLX4720 treatment. We previously found a relatively high background expression level of p21 $1^{\text {Cip1 } 1}$ in A375P/Mdr cells compared to A375P cells [15]. Conversely, PLX4720 treatment only moderately increased the levels of p27 ${ }^{\text {Kip1 }}$ in A375P/Mdr cells. However, miR-1246 had no effect on PLX4720-induced changes in the levels of p21 ${ }^{\mathrm{Cip} 1}$ and p27 ${ }^{\mathrm{Kip} 1}$, which implies that the miR-1246-mediated increase in $\mathrm{G}_{2} / \mathrm{M}$ phase was not mediated by CKIs.

Because chemotherapeutic agents can cause apoptosis after $\mathrm{G}_{2} / \mathrm{M}$ arrest, apoptotic cell death was assessed by staining with FITC-labeled annexin V and PI. The data presented in Fig. $3 \mathrm{C}$ revealed that the primary mechanism of cell death 

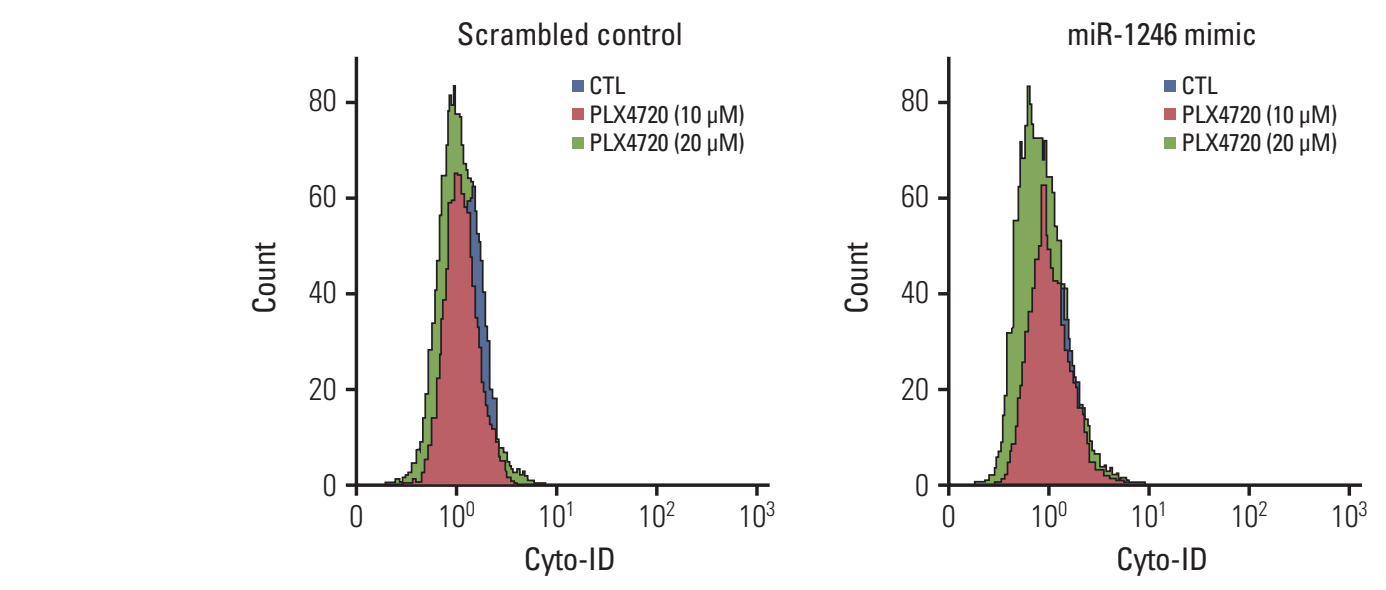

A

B
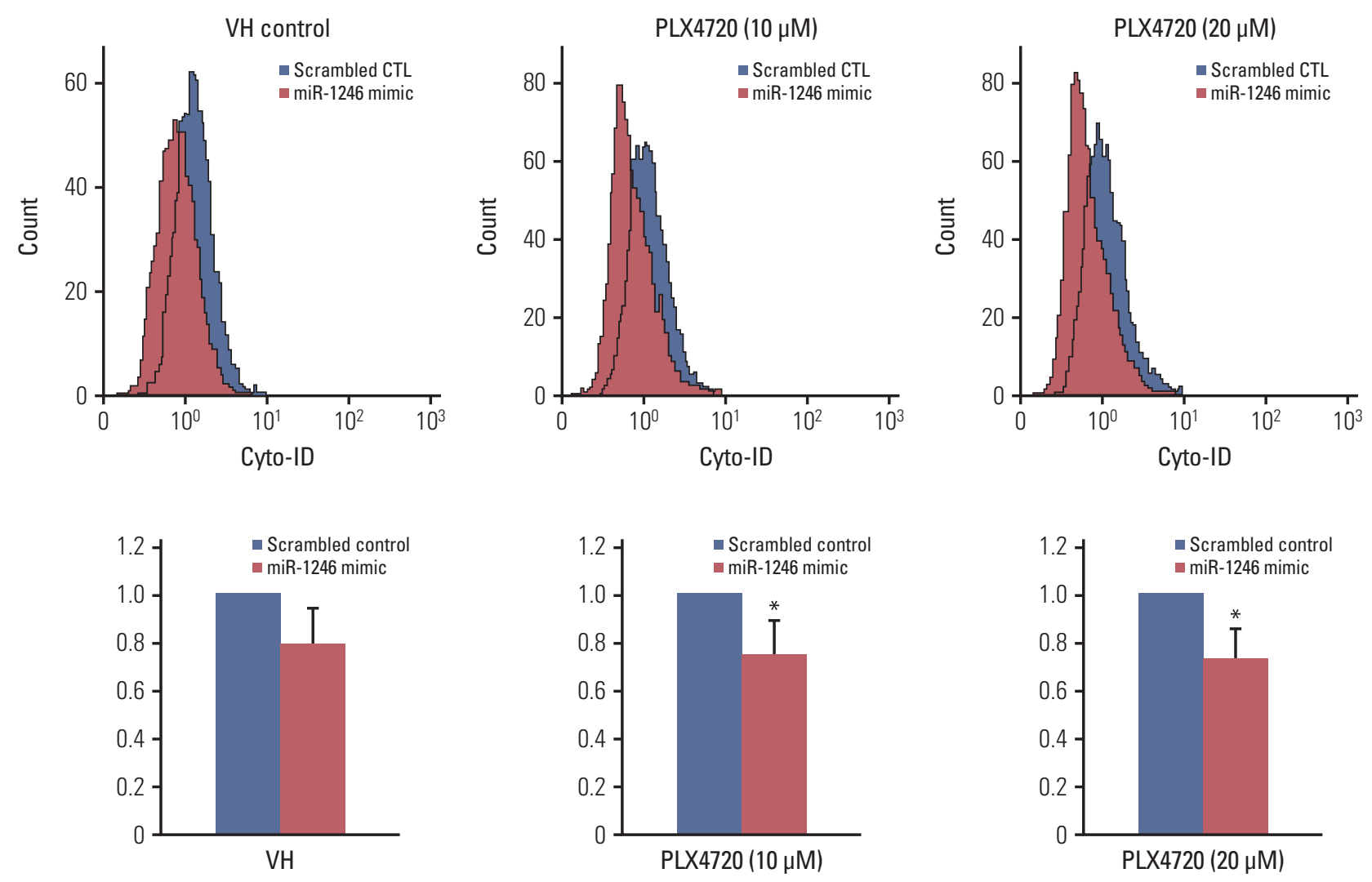

Fig. 4. Impaired autophagic flux during PLX4720-induced growth inhibition of A375P/Mdr cells. After transient transfection with a miR-1246 mimic or control RNA for 24 hours, autophagic flux was monitored in cells exposed to $20 \mu \mathrm{M}$ PLX4720 for 48 hours. Autophagy was then detected by flow cytometric analysis of cells incubated with Cyto-ID green fluorescent probes, which detect autophagic vacuoles. (A) A representative histogram of the flow cytometric analysis is shown. Data are representative of at least two independent experiments. (B) The values are expressed as a fold increase, where the value observed in control RNA-transfected cells was set to 1.0. The upper panel shows a representative histogram of flow cytometric analysis. The lower panel shows the summarized data describing the percentage of cells positive for Cyto-ID fluorescence $(n=3)$. CTL, control. ${ }^{*} \mathrm{p}<0.05$ compared with the vehicle control, as determined by Dunnett's $\mathrm{t}$ test. 


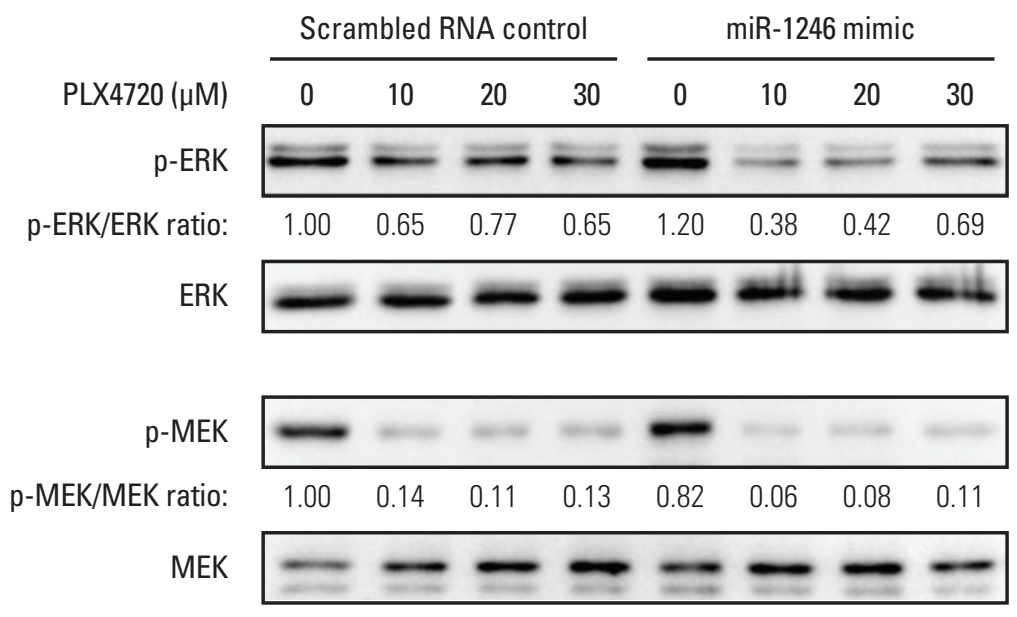

Fig. 5. Effect of miR-1246 on MEK-ERK signaling. A375P/Mdr cells were transiently transfected with a miR-1246 mimic or control RNA for 24 hours. Cell lysates were then prepared after treatment with the indicated concentrations of PLX4720 for 24 hours. The phosphorylated forms of MEK and ERK were detected by immunoblotting using anti-p-MEK and anti-p-ERK antibodies. The same blots were stripped and reprobed with anti-MEK and anti-ERK antibodies to confirm similar expression levels of MEK and ERK proteins in all lanes. The numbers listed below each band indicate the phosphorylated protein/total protein ratios determined using the Image Lab software. Data are representative of at least three independent experiments.

appeared to differ between miR-1246 mimic-transfected and mimic-control-transfected A375P/Mdr cells. For the miR1246 mimic-transfected cells, PLX4720 resulted in the significant emergence of apoptotic cells, but not necrotic cells. In miR-1246 mimic-transfected cells, the necrosis/apoptosis ratio was 0.32 . Conversely, among the mimic-control-transfected cells, the majority died via necrosis instead of apoptosis. The results showed the ratio of necrosis/apoptosis in mimic-control-transfected cells was 4.33 . These data imply that the difference in sensitivity to PLX4720 between mimiccontrol-transfected and miR-1246 mimic-transfected cells may result from the difference in the proportion of the necrotic cell population.

\section{The role of autophagy in miR-1246-mediated resistance to the BRAF inhibitor}

We previously reported that inhibitors of oncogenic BRAF offer an effective therapeutic strategy against A375P BRAFV600E melanoma by modulating autophagy $[12,16]$. Thus, we studied the role of autophagy in PLX4720-induced growth inhibition using miR-1246-transfected cells. The change in autophagy was examined by flow cytometry using Cyto-ID, a green fluorescent dye that accumulates in autophagic vacuoles [14]. This assay was recently demonstrated to be a rapid and quantitative method for monitoring of autophagy [17]. A representative flow cytometry histogram of Cyto-ID fluorescence is shown in Fig. 4A. Treat- ment with PLX4720 resulted in a dose-dependent decrease in autophagy of miR-1246 mimic-transfected cells; however, it did not affect autophagy of mimic-control-transfected cells. Fig. 4B shows that, when compared with mimic-controltransfected cells, PLX4720 significantly decreased Cyto-ID fluorescence intensity in miR-1246-transfected A375P/Mdr cells. These results imply that the miR-1246-induced inhibition of autophagy may contribute to the resistance to BRAF inhibitors.

\section{Effects of miR-1246 on MEK-ERK signaling}

Because it is known that $\mathrm{A} 375 \mathrm{P} / \mathrm{Mdr}$ cells are resistant to BRAF inhibitor-induced ERK inhibition [6], we used immunoblotting to examine phospho-MEK and phosphoERK levels in mimic-control-RNA-transfected and miR1246-transfected A375P/Mdr cells after PLX4720 treatment (Fig. 5). As previously reported, p-ERK levels remained relatively elevated in mimic-control-transfected cells in the presence of PLX4720. Just as in BRAF inhibitor-sensitive A375P cells, marked downregulation of $\mathrm{p}$-ERK was detected in miR-1246-transfected A375P/Mdr cells after PLX4720 treatment. Nonetheless, PLX4720 almost completely abrogated the phosphorylation of MEK, the upstream activator of ERK, in both cell types. These results suggest that the promotion of BRAF inhibitor resistance by miR-1246 was associated with lowered levels of p-ERK. 


\section{Discussion}

The onset of acquired resistance limits clinical efficacy of BRAF inhibitors [4]. Although several studies have explored the roles of miRNAs in melanoma development and progression $[10,18]$, little is known about the possible involvement of microRNAs in acquisition of BRAF inhibitor resistance. Here, we identified 43 miRNAs associated with resistance to BRAF inhibitors using a miRNA array platform. TaqMan quantitative RT-PCR (qRT-PCR) analyses showed that five of these miRNAs (miR-3617, miR-92a1, miR-1246, miR-193b$3 p$, and miR-17-3p) may be associated with resistance to the BRAF inhibitor, confirming the change in expression of these miRNAs in two BRAF inhibitor-resistant cell lines. These five miRNAs have not been extensively studied in terms of resistance to BRAF inhibitors; however, some play oncogenic or tumor suppressor roles in cancers. miR-92a and miR$17-3 p$ are upregulated in tumor cells and associated with increased proliferation and tumorigenesis $[19,20]$. Among the best studied is miR-1246, which was first identified during sequencing of human stem cells in 2008 [21]. Nonetheless, there is an ongoing debate regarding whether miR-1246 acts as an oncomicroRNA or tumor suppressor. Although the expression of miR-1246 has been reported to be negatively correlated with cancer progression [22], many other studies revealed that miR-1246 can enhance cell migration and invasion in cancer cells [23], supporting the oncogenic role of miR-1246. In particular, several reports on miR-1246 have shown that miR-1246 promotes tumorigenesis by targeting cytoplasmic polyadenylation element-binding protein 4 [24] and thrombospondin 2 (THBS2) [25].

Based on microarray data, the average level of miR-1246 was found to be over threefold higher in two BRAF inhibitorresistant cells than in BRAF inhibitor-sensitive cells. A cell growth assay revealed that the miR-1246 mimic confers the strongest resistance to the BRAF inhibitor (PLX4720) in A375P / Mdr cells. In addition, miR-1246 mimic also significantly reduced the antiproliferative effects of PLX4720 in SK-MEL-2 cells, another cell line resistant to BRAF inhibitors; however, the efficacy of miR-1246 was modest. Notably, our miR-1246 mimic failed to reverse the growth inhibition by PLX4720 in BRAF inhibitor-sensitive A375P cells. Thus, these results indicate that a target gene of miR-1246 may be upregulated in cells with acquired resistance to BRAF inhibitors. In agreement with our results suggesting that miR-1246 expression is associated with resistance to anti-BRAF therapy, the in vitro drug sensitivity of pancreatic cancer cells is known to be altered depending on miR-1246 expression [26]. Nevertheless, the exact mechanism of action of miR-1246 in drug resistance is not completely understood. Our previous study revealed that treatment with a BRAF inhibitor leads to a dose-dependent apoptotic changes in BRAF inhibitor-sensitive A375P cells [3]. In the present study, our flow cytometry results clearly showed necrosis instead of apoptosis in BRAF inhibitor-resistant A375P/Mdr cells based on their being $17.87 \%$ PI-positive annexin $\mathrm{V}$-negative cells versus only $4.12 \%$ PI-negative annexin V-positive cells. One possible mechanism behind the higher rate of necrosis in BRAF inhibitor-resistant A375P / Mdr cells may be upregulation of antiapoptotic pathways in A375P/Mdr cells, which may shift the cells to necrosis during PLX4720 treatment. Conversely, miR-1246 mimic transfection induced very little necrosis, but a moderate degree of apoptosis in A375P/Mdr cells. These results indicate that resistance to PLX4720 in miR-1246 mimic-transfected cells is predominantly due to a reduction in necrosis concomitant with a moderate increase in apoptosis. It has been reported that miR-1246 can induce p53dependent apoptosis in response to DNA damage [27].

Nevertheless, autophagy has two seemingly contradictory effects on tumor development. Increasing evidence indicates that autophagy can either promote cell survival at certain stages or cell death at other stages [28]. In our study, PLX4720 produced a significant decrease of autophagy in miR-1246transfected cells; however, it had no inhibitory effect in mimic-control-transfected cells, implying that the miR-1246induced inhibition of autophagy may contribute to resistance to BRAF inhibitors. In fact, $\mathrm{G}_{2} / \mathrm{M}$ arrest is associated with a survival mechanism for cancer cells as a way to escape cell death induced by anticancer therapy [29]. In the present study, we found that PLX4720 caused much more $\mathrm{G}_{2} / \mathrm{M}$ arrest in $\mathrm{A} 375 \mathrm{P} / \mathrm{Mdr}$ cells transfected with the miR-1246 mimic than that seen in mimic-control-RNA-transfected cells. Thus, our results indicate that miR-1246 increases the cell population in the $\mathrm{G}_{2} / \mathrm{M}$ phase through autophagy inhibition and promotes escape from cell death by necrosis and apoptosis in response to PLX4720.

The continued activation of the MAPK pathway observed in the presence of PLX4720 is believed to be a major cause of BRAF resistance in BRAF inhibitor-resistant cells. It has been reported that activation of ERK leads to negative feedback regulation of MEK [30]. Our previous results also showed that long-term treatment with BRAF inhibitors significantly downregulated Spry2 in BRAF V600E-positive cell lines, and that this change is accompanied by rebound activation of the MAPK pathway [6]. As expected, the phospho-ERK levels were relatively unresponsive to PLX4720 in mimic-controltransfected A375P/Mdr cells. Conversely, a decrease in the amount of p-ERK was noted in miR-1246 mimic-transfected A375P/Mdr cells, suggesting that the promotion of BRAF inhibitor resistance by miR-1246 may be associated with lowered levels of $\mathrm{p}$-ERK. Interestingly, in miR-1246 mimic-transfected A375P/Mdr cells, PLX4720 induced the phosphorylation of ERK in a slight dose-dependent manner up to 30 
$\mu \mathrm{M}$. We previously reported that PLX4720 induced paradoxical phosphorylation of ERK in SK-MEL-2 cells that showed a much lower level of $\mathrm{p}$-ERK compared to A375P cells with elevated levels of p-ERK [3]. Thus, in this study, we cannot exclude the possibility that, as in SK-MEL-2 cells, PLX4720 induced the paradoxical activation of ERK in A375P/Mdr cells in which p-ERK was markedly downregulated by miR1246. These data point to another mechanism behind the dysregulation of the MAPK pathway that may contribute to the profound resistance associated with current BRAF-targeted therapies.

Taken together, our results indicate that miR-1246 may be a potential therapeutic target in melanoma with acquired resistance to BRAF inhibitors. Nonetheless, the mechanism underlying the miR-1246-induced resistance to the BRAF inhibitor is unknown. Thus, a better understanding of miR1246 regulation should advance our knowledge of the molecular mechanisms behind resistance to chemotherapy. Further research investigating a miR-1246 knockout model is needed to identify the potential target genes of miR-1246 and fully evaluate the functional impact and importance of miR-1246 in melanoma cells.

\section{Electronic Supplementary Material}

Supplementary materials are available at Cancer Research and Treatment website (http://www.e-crt.org).

\section{Conflicts of Interest}

Conflict of interest relevant to this article was not reported.

\section{Acknowledgments}

This research was supported by the Basic Science Research Program through the National Research Foundation of Korea (NRF) funded by the Ministry of Education, Science and Technology (2016R1D1A1B03930193) and by the Incheon National University Research Grant in 2014.

\section{References}

1. Davies H, Bignell GR, Cox C, Stephens P, Edkins S, Clegg S, et al. Mutations of the BRAF gene in human cancer. Nature. 2002;417:949-54.

2. Holderfield M, Deuker MM, McCormick F, McMahon M. Targeting RAF kinases for cancer therapy: BRAF-mutated melanoma and beyond. Nat Rev Cancer. 2014;14:455-67.

3. Kim YK, Ahn SK, Lee M. Differential sensitivity of melanoma cell lines with differing B-Raf mutational status to the new oncogenic B-Raf kinase inhibitor UI-152. Cancer Lett. 2012;320:215-24.

4. Rizos H, Menzies AM, Pupo GM, Carlino MS, Fung C, Hyman $\mathrm{J}$, et al. BRAF inhibitor resistance mechanisms in metastatic melanoma: spectrum and clinical impact. Clin Cancer Res. 2014;20:1965-77.

5. Johannessen CM, Boehm JS, Kim SY, Thomas SR, Wardwell $\mathrm{L}$, Johnson LA, et al. COT drives resistance to RAF inhibition through MAP kinase pathway reactivation. Nature. 2010;468: 968-72.

6. Ahn JH, Han BI, Lee M. Induction of resistance to BRAF inhibitor is associated with the inability of Spry2 to inhibit BRAF-V600E activity in BRAF mutant cells. Biomol Ther (Seoul). 2015;23:320-6.

7. Chandarlapaty S. Negative feedback and adaptive resistance to the targeted therapy of cancer. Cancer Discov. 2012;2: 311-9.

8. Bartel DP. MicroRNAs: genomics, biogenesis, mechanism, and function. Cell. 2004;116:281-97.

9. Ma J, Dong C, Ji C. MicroRNA and drug resistance. Cancer Gene Ther. 2010;17:523-31.

10. Pinto R, Strippoli S, De Summa S, Albano A, Azzariti A, Guida $\mathrm{G}$, et al. MicroRNA expression in BRAF-mutated and wildtype metastatic melanoma and its correlation with response duration to BRAF inhibitors. Expert Opin Ther Targets. 2015;19:1027-35.

11. Stark MS, Bonazzi VF, Boyle GM, Palmer JM, Symmons J, Lanagan CM, et al. miR-514a regulates the tumour suppressor NF1 and modulates BRAFi sensitivity in melanoma. Oncotarget. 2015;6:17753-63.

12. Ahn JH, Lee M. Autophagy-dependent survival of mutant B-Raf melanoma cells selected for resistance to apoptosis induced by inhibitors against oncogenic B-Raf. Biomol Ther (Seoul). 2013;21:114-20.

13. Livak KJ, Schmittgen TD. Analysis of relative gene expression data using real-time quantitative PCR and the 2(-Delta Delta C(T)) Method. Methods. 2001;25:402-8.

14. Oeste CL, Seco E, Patton WF, Boya P, Perez-Sala D. Interactions between autophagic and endo-lysosomal markers in endothelial cells. Histochem Cell Biol. 2013;139:659-70.

15. Jang GH, Kim NY, Lee M. Low inducible expression of p21Cip1 confers resistance to paclitaxel in BRAF mutant melanoma cells with acquired resistance to BRAF inhibitor. Mol Cell Biochem. 2015;406:53-62. 
16. Ahn JH, Lee YW, Ahn SK, Lee M. Oncogenic BRAF inhibitor UAI-201 induces cell cycle arrest and autophagy in BRAF mutant glioma cells. Life Sci. 2014;104:38-46.

17. Lee JS, Lee GM. Monitoring of autophagy in Chinese hamster ovary cells using flow cytometry. Methods. 2012;56:375-82.

18. Segura MF, Greenwald HS, Hanniford D, Osman I, Hernando E. MicroRNA and cutaneous melanoma: from discovery to prognosis and therapy. Carcinogenesis. 2012;33:1823-32.

19. Mishra RR, Kneitz S, Schartl M. Comparative analysis of melanoma deregulated miRNAs in the medaka and Xiphophorus pigment cell cancer models. Comp Biochem Physiol C Toxicol Pharmacol. 2014;163:64-76.

20. Zhu J, Dong H, Zhang Q, Zhang S. Combined assays for serum carcinoembryonic antigen and microRNA-17-3p offer improved diagnostic potential for stage I/II colon cancer. Mol Clin Oncol. 2015;3:1315-8.

21. Bar M, Wyman SK, Fritz BR, Qi J, Garg KS, Parkin RK, et al. MicroRNA discovery and profiling in human embryonic stem cells by deep sequencing of small RNA libraries. Stem Cells. 2008;26:2496-505.

22. Zhang Q, Cao LY, Cheng SJ, Zhang AM, Jin XS, Li Y. p53induced microRNA-1246 inhibits the cell growth of human hepatocellular carcinoma cells by targeting NFIB. Oncol Rep. 2015;33:1335-41

23. Sun Z, Meng C, Wang S, Zhou N, Guan M, Bai C, et al. MicroRNA-1246 enhances migration and invasion through
CADM1 in hepatocellular carcinoma. BMC Cancer. 2014; 14:616.

24. Huang W, Li H, Luo R. The microRNA-1246 promotes metastasis in non-small cell lung cancer by targeting cytoplasmic polyadenylation element-binding protein 4. Diagn Pathol. 2015;10:127.

25. Chen J, Yao D, Zhao S, He C, Ding N, Li L, et al. MiR-1246 promotes SiHa cervical cancer cell proliferation, invasion, and migration through suppression of its target gene thrombospondin 2. Arch Gynecol Obstet. 2014;290:725-32.

26. Hasegawa S, Eguchi H, Nagano H, Konno M, Tomimaru Y, Wada $\mathrm{H}$, et al. MicroRNA-1246 expression associated with CCNG2-mediated chemoresistance and stemness in pancreatic cancer. Br J Cancer. 2014;111:1572-80.

27. Zhang Y, Liao JM, Zeng SX, Lu H. p53 downregulates Down syndrome-associated DYRK1A through miR-1246. EMBO Rep. 2011;12:811-7.

28. Eisenberg-Lerner A, Bialik S, Simon HU, Kimchi A. Life and death partners: apoptosis, autophagy and the cross-talk between them. Cell Death Differ. 2009;16:966-75.

29. Kawabe T. G2 checkpoint abrogators as anticancer drugs. Mol Cancer Ther. 2004;3:513-9.

30. Roskoski R Jr. MEK1/ 2 dual-specificity protein kinases: structure and regulation. Biochem Biophys Res Commun. 2012; 417:5-10. 